\title{
Local existence of polynomial decay solutions to the Boltzmann equation for soft potentials
}

AUTHOR(S):

Morimoto, Yoshinori; Yang, Tong

\section{CITATION:}

Morimoto, Yoshinori ... [et al]. Local existence of polynomial decay solutions to the Boltzmann equation for soft potentials. Analysis and Applications 2015, 13(06): 663-683

\section{ISSUE DATE:}

2015-11

URL:

http://hdl.handle.net/2433/202522

\section{RIGHT:}

Electronic version of an article published as [Anal. Appl. 13, 663 (2015). DOI: 10.1142/S0219530514500079] @ World Scientific Publishing Company; The full-text file will be made open to the public on 31 December 2016 in accordance with publisher's 'Terms and Conditions for Self-Archiving'.; This is not the published version. Please cite only the published version.; この論文は出版社版でありません。引用の際には出版社版をご確認ご利用ください。 


\title{
LOCAL EXISTENCE OF POLYNOMIAL DECAY SOLUTIONS TO THE BOLTZMANN EQUATION FOR SOFT POTENTIALS
}

\author{
YOSHINORI MORIMOTO AND TONG YANG
}

\begin{abstract}
The existence of classical solutions to the Cauchy problem for the Boltzmann equation without angular cutoff has been extensively studied in the framework when the solution has Maxwellian decay in the velocity variable. cf. $[8,6]$ and the references therein. In this paper, we prove local existence of solutions with polynomial decay in the velocity variable for the Boltzmann equation with soft potential. In the proof, the singular change of variables between post- and pre-collision velocities plays an important role, as well as the regular one introduced in the celebrated cancellation lemma by AlexandreDesvillettes-Villani-Wennberg [1].
\end{abstract}

\section{INTRODUCTION}

Consider the Cauchy problem for the Boltzmann equation,

$$
\partial_{t} f+v \cdot \nabla_{x} f=Q(f, f), \quad f(0, x, v)=f_{0}(x, v),
$$

where $f=f(t, x, v)$ is the density distribution function of particles with velocity $v \in \mathbb{R}^{3}$ at time $t$ and position $x$. The right hand side of (1.1) is given by the Boltzmann bilinear collision operator

$$
Q(g, f)(v)=\int_{\mathbb{R}^{3}} \int_{\mathbb{S}^{2}} B\left(v-v_{*}, \sigma\right)\left\{g\left(v_{*}^{\prime}\right) f\left(v^{\prime}\right)-g\left(v_{*}\right) f(v)\right\} d \sigma d v_{*},
$$

which is well-defined for suitable functions $f$ and $g$, specified later. Notice that the collision operator $Q(\cdot, \cdot)$ acts only on the velocity variable $v \in \mathbb{R}^{3}$. In the following discussion, we will use the $\sigma$-representation, that is, for $\sigma \in \mathbb{S}^{2}$,

$$
v^{\prime}=\frac{v+v_{*}}{2}+\frac{\left|v-v_{*}\right|}{2} \sigma, v_{*}^{\prime}=\frac{v+v_{*}}{2}-\frac{\left|v-v_{*}\right|}{2} \sigma,
$$

which follows from the conservation of the moment and energy in the elastic collision. The non-negative cross section $B(z, \sigma)$ depends only on $|z|$ and the scalar product $\frac{z}{|z|} \cdot \sigma$. From the consideration of physical models, it usually takes the form

$$
B\left(\left|v-v_{*}\right|, \cos \theta\right)=\Phi\left(\left|v-v_{*}\right|\right) b(\cos \theta), \quad \cos \theta=\frac{v-v_{*}}{\left|v-v_{*}\right|} \cdot \sigma, \quad 0 \leq \theta \leq \frac{\pi}{2},
$$

where

$$
\begin{aligned}
& \Phi(|z|)=\Phi_{\gamma}(|z|)=|z|^{\gamma}, \text { for some } \gamma>-3, \\
& b(\cos \theta) \theta^{2+2 s} \rightarrow K \text { when } \theta \rightarrow 0+\text {, for } 0<s<1 \text { and } K>0 .
\end{aligned}
$$

In fact, if the inter-molecule potential satisfies the inverse power law with potential being $U(\rho)=\rho^{-(q-1)}, q>2$, where $\rho$ denotes the distance between the two 
interacting molecules, then $s$ and $\gamma$ are given by

$$
0<s=1 /(q-1)<1, \quad 1>\gamma=1-4 s=(q-5) /(q-1)>-3 .
$$

As usual, the hard $(\gamma>0)$ and soft $(\gamma<0)$ potentials correspond to $q>5$ and $2<q<5$, respectively, and the Maxwellian potential $(\gamma=0)$ corresponds to $q=5$.

The angle $\theta$ is the deviation angle, i.e., the angle between post- and pre-collisional velocities. Though the range of $\theta$ is originally the interval $[0, \pi]$, it should be noted that the angle $\theta$ in (1.2) is now restricted to $[0, \pi / 2]$, as in [1], by replacing $b(\cos \theta)$ by its "symmetrized" version

$$
[b(\cos \theta)+b(\cos (\pi-\theta))] \mathbf{1}_{0 \leq \theta \leq \pi / 2},
$$

which is possible due to the invariance of the product $f\left(v^{\prime}\right) f\left(v_{*}^{\prime}\right)$ in the collision operator $Q(f, f)$ under the change of variables $\sigma \rightarrow-\sigma$. This enables us to use the regular change of variables $v \rightarrow v^{\prime}$ between post- and pre-collisional velocities ( see the proof of the cancellation lemma in [1] and (2.3) below).

The singular change of variables $v_{*} \rightarrow v^{\prime}$ was firstly introduced in [9, 2] to show the existence of solutions to the linearized Boltzmann equation, and was used in $[3,4,7]$ to show the uniqueness of solutions with polynomial decay in the velocity variable to the nonlinear Boltzmann equation for Maxwellian and soft potentials.

The purpose of the present paper is to give a local existence result concerning polynomial decay solutions in the velocity variable to the nonlinear Boltzmann equation for certain soft potentials. Namely, because of the technical difficulties, we confine ourselves to the case

$$
0<s<\frac{1}{2},-\frac{3}{2}<\gamma \leq 0
$$

though the uniqueness of solutions was discussed in [7] under a more general condition that requires $0<s<1$ and $\max \{-3,-2 s-3 / 2\}<\gamma \leq 0$.

We introduce the function space for the solutions as follows. Set

$$
\partial_{\beta}^{\alpha}=\partial_{x}^{\alpha} \partial_{v}^{\beta}, \quad \alpha, \beta \in \mathbb{N}^{3},
$$

and

$$
\mathcal{W}= \begin{cases}\langle v\rangle & \text { if } 0<s \leq 1 / 4 \\ \langle v\rangle^{2 s /(1-2 s)} & \text { if } 1 / 4<s<1 / 2\end{cases}
$$

which ensures $\langle v\rangle \leq \min \left\{\mathcal{W}^{\frac{1-2 s}{2 s}}, \mathcal{W}\right\}$ for the later use. As in [5], we use a weight function in both the space and velocity variables

$$
\phi(x, v)=\frac{1}{1+|v|^{2}+|x|^{2}},
$$

which possesses the commutator property $\left|\left[v \cdot \nabla_{x}, \phi\right]\right|=2|v \cdot x| \phi^{2} \leq \phi$. For $k \in \mathbb{N}$, $\ell \in \mathbb{R}$ with $k<\ell$, we define

$$
\begin{aligned}
\mathcal{H}_{u l}^{k, \ell} & \left(\mathbb{R}^{6}\right)=\left\{g \mid\|g\|_{\mathcal{H}_{u l}^{k, \ell}\left(\mathbb{R}^{6}\right)}^{2}\right. \\
& \left.=\sum_{|\alpha+\beta| \leq k} \sup _{a \in \mathbb{R}^{3}} \int_{\mathbb{R}^{6}}\left|\phi(x-a, v) \mathcal{W}^{\ell-|\alpha+\beta|} \partial_{\beta}^{\alpha} g(x, v)\right|^{2} d x d v<+\infty\right\} .
\end{aligned}
$$

The function space $\mathcal{H}_{u l}^{k, \ell}\left(\mathbb{R}^{6}\right)$ is a variant of the uniformly local Sobolev space $H_{u l}^{k, \ell}\left(\mathbb{R}^{6}\right)$ used in [6], which is defined by replacing $\mathcal{W}^{\ell-|\alpha+\beta|}$ and $\phi(x, v)$ by $\langle v\rangle^{\ell}$ and a usual smooth cutoff function $\phi_{1}(x) \in C_{0}^{\infty}\left(\mathbb{R}^{3}\right)$, respectively. In [6], bounded classical solution with Maxwellian decay in the velocity variable is constructed in 
the whole space without specifying any limit behavior at the spatial infinity and without assuming the smallness condition on initial data, under the assumption $0<s<1 / 2,-3 / 2<\gamma, \gamma+2 s<1$ on the cross section $B$.

Now for the local existence of polynomial decay solution in the velocity variable, we have the following improvement of Theorem 1.1 of [6] for the soft potentials.

Theorem 1.1. Assume that the cross section $B$ takes the form (1.2) and satisfies (1.3). If the initial data $f_{0}$ is non-negative and belongs to $\mathcal{H}_{u l}^{k, \ell}\left(\mathbb{R}^{6}\right)$ for $k \geq 6$ and $\ell \geq k+7$, then there exists a $T_{*}>0$ such that the Cauchy problem (1.1) admits a non-negative unique solution in the function space $C^{0}\left(\left[0, T_{*}\right] ; \mathcal{H}_{u l}^{k, \ell}\left(\mathbb{R}^{6}\right)\right)$.

Throughout this paper, we will use the following notation: $A \lesssim B$ means that there exists a generic positive constant $C>0$ such that $A \leq C B$. Furthermore, $A \sim B$ means that $A \lesssim B$ and $B \lesssim A$.

The rest of the paper will be organized as follows. In the next section, we will present some preliminary lemmas, in particular, including an estimate about how to compensate the order of moment and the order of differentiation that is one of the key observations in the analysis. The uniform estimate based on the estimations on the commutators and the collision operator will be given in Section 3. In Section 4, we study the cutoff approximations for the construction of local solutions. Finally, the technical estimation on the gain part of the collision operator will be given in the last section.

\section{PRELIMINARY LEMMAS}

First we prepare a lemma concerning the interpolation of moments and derivatives.

Lemma 2.1. Let $m>0$ and $0<\delta<1$. Then for any $f \in \mathcal{S}\left(\mathbb{R}^{n}\right)$, we have

$$
\left\|\langle v\rangle^{-m \delta}\left\langle D_{v}\right\rangle^{\delta} f\right\| \lesssim\|f\|+\left\|\langle v\rangle^{-m}\left\langle D_{v}\right\rangle f\right\| .
$$

Proof. Take the Paley-Littlewood decomposition

$$
\sum_{j=0}^{\infty} \varphi_{j}(v)=1, \quad \varphi_{0} \in C_{0}^{\infty}, \varphi_{j}(v)=\varphi\left(2^{-j} v\right) \text { for } j \geq 1,
$$

where $\varphi \in C_{0}^{\infty}$ with $\operatorname{supp} \varphi \subset\{1 \leq|v| \leq 2\}$. It follows from the locally finite covering property that

$$
\begin{aligned}
\left\|\langle v\rangle^{-m \delta} \sum_{j=0}^{\infty} \varphi_{j}\left\langle D_{v}\right\rangle^{\delta} f\right\|^{2} & \lesssim \sum_{j=0}^{\infty}\left\|\langle v\rangle^{-m \delta} \varphi_{j}\left\langle D_{v}\right\rangle^{\delta} f\right\|^{2} \\
& \lesssim \sum_{j=0}^{\infty} 4^{-m j \delta}\left\|\varphi_{j}\left\langle D_{v}\right\rangle^{\delta} f\right\|^{2} \\
& \lesssim \sum_{j=0}^{\infty}\left(4^{-m j \delta}\left\|\left\langle D_{v}\right\rangle^{\delta} \varphi_{j} f\right\|^{2}+4^{-m j \delta}\left\|\left[\varphi_{j},\left\langle D_{v}\right\rangle^{\delta}\right] f\right\|^{2}\right) \\
& =\sum_{j=0}^{\infty} I_{j}+\sum_{j=0}^{\infty} R_{j} .
\end{aligned}
$$


Since $\left[\varphi_{j},\left\langle D_{v}\right\rangle^{\delta}\right]$ is a $L^{2}$ bounded operator uniformly with respect to $j$, we have

$$
\sum R_{j} \lesssim\|f\|^{2} \sum 4^{-m j \delta} \lesssim\|f\|^{2} .
$$

Noting $\left(\frac{\langle\xi\rangle^{2}}{4^{m j}}\right)^{\delta} \leq 1+\frac{\langle\xi\rangle^{2}}{4^{m j}}$, we get

$$
\begin{aligned}
I_{j} & \leq\left\|\varphi_{j} f\right\|^{2}+4^{-m j}\left\|\left\langle D_{v}\right\rangle \varphi_{j} f\right\|^{2} \\
& \lesssim\left\|\varphi_{j} f\right\|^{2}+4^{-m j}\left\|\varphi_{j}\left\langle D_{v}\right\rangle f\right\|^{2}+4^{-m j}\left\|\left[\left\langle D_{v}\right\rangle, \varphi_{j}\right] f\right\|^{2},
\end{aligned}
$$

from which we have $\sum I_{j} \lesssim\|f\|^{2}+\left\|\langle v\rangle^{-m}\left\langle D_{v}\right\rangle f\right\|^{2}$ because

$$
\sum_{j=0}^{\infty} \varphi_{j}^{2} \lesssim 1, \sum_{j=0}^{\infty} 4^{-m j} \varphi_{j}^{2} \lesssim\langle v\rangle^{-2 m} .
$$

Lemma 2.2. Let $\phi(x, v)$ be the function defined in (1.5). Then we have

$$
\begin{aligned}
\|f\|_{L^{\infty}\left(\mathbb{R}_{x}^{3} ; L^{2}\left(\mathbb{R}_{v}\right)\right)} & \lesssim \sum_{|\alpha| \leq 2} \sup _{a \in \mathbb{R}_{x}^{3}}\left\|\phi(x-a, v)\langle v\rangle^{2} \partial_{x}^{\alpha} f\right\|_{L^{2}\left(\mathbb{R}^{6}\right)} \\
& \sim \sup _{a \in \mathbb{R}_{x}^{3}}\left\|\left(1-\Delta_{x}\right) \phi(x-a, v)\langle v\rangle^{2} f\right\|_{L^{2}\left(\mathbb{R}^{6}\right) .}
\end{aligned}
$$

Proof. If we denote $g(x, v ; a)=\langle v\rangle^{2} f(x, v) /\left(1+|v|^{2}+|x-a|^{2}\right)$, then it follows from the Sobolev embedding that

$$
\begin{aligned}
|f(a, v)|^{2} & \leq\|g(\cdot, v ; a)\|_{L_{x}^{\infty}}^{2} \lesssim\left\|\left(1-\Delta_{x}\right) g(\cdot, v ; a)\right\|_{L_{x}^{2}}^{2} \\
& \lesssim \sum_{|\alpha| \leq 2} \int_{\mathbb{R}_{x}^{3}}\left|\phi(x-a, v)\langle v\rangle^{2} \partial_{x}^{\alpha} f(x, v)\right|^{2} d x .
\end{aligned}
$$

Here the last inequality follows from $\left|\partial_{x}^{\alpha} \phi\right| \lesssim \phi$. Integrating both sides with respect to $v$, we obtain

$$
\begin{aligned}
\int_{\mathbb{R}_{v}^{3}}|f(a, v)|^{2} d v & \lesssim \sum_{|\alpha| \leq 2} \int_{\mathbb{R}^{6}}\left|\phi(x-a, v)\langle v\rangle^{2} \partial_{x}^{\alpha} f(x, v)\right|^{2} d x d v \\
& \lesssim \sum_{|\alpha| \leq 2} \sup _{a^{\prime} \in \mathbb{R}_{x}^{3}} \int_{\mathbb{R}^{6}}\left|\phi\left(x-a^{\prime}, v\right)\langle v\rangle^{2} \partial_{x}^{\alpha} f(x, v)\right|^{2} d x d v
\end{aligned}
$$

which gives the desired estimate because $a \in \mathbb{R}^{3}$ is arbitrary.

For the estimation on $\left\|Q\left(\partial^{k} f, \phi_{a} \mathcal{W}^{\ell-k} f\right)\right\|_{L^{2}\left(\mathbb{R}^{6}\right)}$, we need the following

Lemma 2.3. Denote $\phi_{a}(x, v)=\phi(x-a, v)$. Then we have

$$
\begin{aligned}
\int \| & f(x, \cdot)\left\|_{L^{2}\left(\mathbb{R}_{v}^{3}\right)}^{2}\right\| \phi_{a}(x, \cdot) g(x, \cdot) \|_{L^{2}\left(\mathbb{R}_{v}^{3}\right)}^{2} d x \\
& \lesssim \int\left\|\phi_{a}(x, \cdot) f(x, \cdot)\right\|_{L_{2}^{2}\left(\mathbb{R}_{v}^{3}\right.}^{2}\|g(x, \cdot)\|_{L^{2}\left(\mathbb{R}_{v}^{3}\right)}^{2} d x \\
& \lesssim\left\|\phi_{a}\langle v\rangle^{2} f\right\|_{L^{2}\left(\mathbb{R}^{6}\right)}^{2}\|g\|_{L^{\infty}\left(\mathbb{R}_{x}^{3} ; L^{2}\left(\mathbb{R}_{v}\right)\right)}^{2} .
\end{aligned}
$$


Proof. This is a direct consequence of

$$
\begin{aligned}
f\left(x, v_{*}\right) \phi_{a}(x, v) g(x, v)= & \left\langle v_{*}\right\rangle^{2} \phi_{a}\left(x, v_{*}\right) f\left(x, v_{*}\right) \phi_{a}(x, v) g(x, v) \\
& +\phi_{a}\left(x, v_{*}\right) f\left(x, v_{*}\right)\left(|x-a|^{2} \phi_{a}(x, v)\right) g(x, v) .
\end{aligned}
$$

For the weight $W_{\phi, \ell}(v)=\phi(x, v)\langle v\rangle^{\ell}$, we first recall Lemma 3.1 and Remark 3.2 of [7] summarized as follows.

Lemma 2.4. For $\ell \geq 4$, we have

$$
\left|W_{\phi, \ell}(v)-W_{\phi, \ell}\left(v^{\prime}\right)\right| \lesssim \frac{\theta\langle v\rangle^{\ell}\left\langle v_{*}\right\rangle^{3}+\theta^{\ell-2}\left\langle v_{*}\right\rangle^{\ell}}{1+|v|^{2}+\left|v_{*}\right|^{2}+|x|^{2}} .
$$

For the commutator of $W_{\phi, \ell}(v)$ and the collision operator, we have

Lemma 2.5. If $-3 / 2<\gamma \leq 0,0<s<1 / 2$ and $l>\max \left\{\frac{7}{2}+2 s, 4\right\}$, then we have

$$
\begin{aligned}
& \left|\left(\left(W_{\phi, \ell} Q(f, g)-Q\left(f, W_{\phi, \ell} g\right)\right), h\right)_{L^{2}\left(\mathbb{R}_{v}^{3}\right)}\right| \\
& \lesssim\|h\|_{L^{2}\left(\mathbb{R}_{v}^{3}\right)}\left(\min \left\{\|\phi f\|_{L_{5}^{2}\left(\mathbb{R}_{v}^{3}\right)}\|g\|_{L_{\ell}^{2}\left(\mathbb{R}_{v}^{3}\right)},\|f\|_{L_{5}^{2}\left(\mathbb{R}_{v}^{3}\right)}\|\phi g\|_{L_{\ell}^{2}\left(\mathbb{R}_{v}^{3}\right)}\right\}\right. \\
& \left.\quad+\min \left\{\|\phi f\|_{L_{\ell}^{2}\left(\mathbb{R}_{v}^{3}\right)}\|g\|_{L_{2}^{2}\left(\mathbb{R}_{v}^{3}\right)},\|f\|_{L_{\ell}^{2}\left(\mathbb{R}_{v}^{3}\right)}\|\phi g\|_{L_{2}^{2}\left(\mathbb{R}_{v}^{3}\right)}\right\}\right),
\end{aligned}
$$

by regarding $x$ in $\phi$ as a parameter.

Proof. It follows from (2.1) that

$$
\begin{aligned}
& \left|\left(W_{\phi, \ell} Q(f, g)-Q\left(f, W_{\phi, \ell} g\right), h\right)_{L^{2}}\right| \\
& \lesssim \iiint\left|v-v_{*}\right|^{\gamma} \mathbf{1}_{\left|v-v_{*}\right| \leq 1} b \theta\left|\left\langle v_{*}\right\rangle^{3} f_{*}\right|\left|\langle v\rangle^{\ell} g\right|\left|h^{\prime}\right| \min \left\{\phi_{*}, \phi\right\} d v d v_{*} d \sigma \\
& \quad+\iiint b \theta\left|\left\langle v_{*}^{\prime}\right\rangle^{3} f_{*}^{\prime}\right|\left|\left\langle v^{\prime}\right\rangle^{\ell} g^{\prime}\right| \min \left\{\phi_{*}^{\prime}, \phi^{\prime}\right\}|h| d v d v_{*} d \sigma \\
& \quad+\iiint b \theta^{\ell-2}\left|\left\langle v_{*}^{\prime}\right\rangle^{\ell} f_{*}^{\prime}\right|\left|g^{\prime}\right| \min \left\{\phi_{*}^{\prime}, \phi^{\prime}\right\}|h| d v d v_{*} d \sigma \\
& =B_{1}+B_{2}+B_{3},
\end{aligned}
$$

where $\phi_{*}=\phi\left(x, v_{*}\right), \phi^{\prime}=\phi\left(x, v^{\prime}\right)$ and so on. By the Cauchy-Schwarz inequality, we have

$$
\begin{aligned}
B_{1}^{2} \lesssim & \iiint\left|v-v_{*}\right|^{\gamma} \mathbf{1}_{\left|v-v_{*}\right| \leq 1} b \theta\left|\left\langle v_{*}\right\rangle^{3} \tilde{f}_{*}\right|\left|\langle v\rangle^{\ell} \tilde{g}\right|^{2} d v d v_{*} d \sigma \\
& \times \iiint\left|v^{\prime}-v_{*}\right|^{\gamma} \mathbf{1}_{\left|v^{\prime}-v_{*}\right| \leq 2} b \theta\left|\left\langle v_{*}\right\rangle^{3} \tilde{f}_{*}\right|\left|h^{\prime}\right|^{2} d v d v_{*} d \sigma \\
= & B_{11} \times B_{12},
\end{aligned}
$$

where $(\tilde{f}, \tilde{g})$ equals $(f, \phi g)$ or $(\phi f, g)$. Since it follows from $0<s<1 / 2$ that $\int b \theta d \sigma<\infty$, we have

$$
\begin{aligned}
B_{11} & \lesssim \int\left(\int\left|v-v_{*}\right|^{2 \gamma} \mathbf{1}_{\left|v-v_{*}\right| \leq 1} d v_{*} \int\left|\left\langle v_{*}\right\rangle^{3} \tilde{f}_{*}\right|^{2} d v_{*}\right)^{1 / 2}\left|\langle v\rangle^{\ell} \tilde{g}\right|^{2} d v \\
& \lesssim\|\tilde{f}\|_{L_{3}^{2}}\|\tilde{g}\|_{L_{\ell}^{2}}^{2},
\end{aligned}
$$


because $\gamma>-3 / 2$. The estimation on $B_{12}$ is obtained with the help of the regular change of variables

$$
v \mapsto v^{\prime}=\frac{v+v_{*}}{2}+\frac{\left|v-v_{*}\right|}{2} \sigma,
$$

which was introduced in [1]. Note that for this change of variables, the Jacobian satisfies

$$
\left|\frac{\partial v}{\partial v^{\prime}}\right|=\frac{8}{|I+\mathbf{k} \otimes \sigma|}=\frac{8}{|1+\mathbf{k} \cdot \sigma|}=\frac{4}{\cos ^{2}(\theta / 2)} \leq 8, \quad \theta \in\left[0, \frac{\pi}{2}\right] .
$$

As in [1], note that after this change of variables, $\mathbf{k}=\left(v-v_{*}\right) /\left|v-v_{*}\right|$ is a function of $v_{*}, v^{\prime}, \sigma$ so that $\theta$ no longer plays the role of polar angle because the "pole" $k$ moves with $\sigma$ so that the surface measure $d \sigma$ is no longer given by $\sin \theta d \theta d \phi$. Therefore, we need a new pole which is independent of $\sigma$ to carry out the integration in $\sigma$. A possible (and indeed the best) choice is $\mathbf{k}^{\prime}=\left(v^{\prime}-v_{*}\right) /\left|v^{\prime}-v_{*}\right|$, for which the polar angle $\psi$ defined by $\cos \psi=k^{\prime} \cdot \sigma$ satisfies (cf. [1, Fig. 1]),

$$
\psi=\frac{\theta}{2}, \quad d \sigma=\sin \psi d \psi d \phi, \quad \psi \in\left[0, \frac{\pi}{4}\right] .
$$

This implies that $\theta$ works almost as polar angle and we can write

$$
B_{12} \lesssim \int_{\mathbb{R}^{3}}\left(\int_{\mathbb{R}^{3}}\left|v^{\prime}-v_{*}\right|^{\gamma} \mathbf{1}_{\left|v^{\prime}-v_{*}\right| \leq 2}\left|\left\langle v_{*}\right\rangle^{3} \tilde{f}_{*}\right| D_{0}\left(v_{*}, v^{\prime}\right) d v_{*}\right)\left|h^{\prime}\right|^{2} d v^{\prime},
$$

with

$$
\begin{aligned}
D_{0}\left(v_{*}, v^{\prime}\right) & =\int_{S^{2}} \theta\left(v_{*}, v^{\prime}, \sigma\right) b\left(\cos \theta\left(v_{*}, v^{\prime}, \sigma\right)\right) d \sigma \\
& \lesssim \int_{0}^{\pi / 4} \psi b(\cos 2 \psi) \sin \psi d \psi<+\infty
\end{aligned}
$$

This deduces $B_{12} \lesssim\|\tilde{f}\|_{L_{3}^{2}}\|h\|_{L^{2}}^{2}$, and then

$$
\left|B_{1}\right| \lesssim\|\tilde{f}\|_{L_{3}^{2}}\|\tilde{g}\|_{L_{\ell}^{2}}\|h\|_{L^{2}}
$$

Using the regular change of variables $v \rightarrow v^{\prime}$ again, we have

$$
\left|B_{2}\right| \lesssim\|\tilde{f}\|_{L_{3}^{1}}\|\tilde{g}\|_{L_{\ell}^{2}}\|h\|_{L^{2}} \lesssim\|\tilde{f}\|_{L_{5}^{2}}\|\tilde{g}\|_{L_{\ell}^{2}}\|h\|_{L^{2}}
$$

by the almost same procedure. As for $B_{3}$, we firstly have

$$
\begin{aligned}
B_{3}^{2}= & \left(\iiint b \theta^{\ell-2}\left|\left\langle v_{*}\right\rangle^{\ell} \tilde{f}_{*}\right||\tilde{g}|\left|h^{\prime}\right| d v d v_{*} d \sigma\right)^{2} \\
\leq & \iiint b \theta^{\ell-2-\frac{3}{2}}|\tilde{g}|\left|\left\langle v_{*}\right\rangle^{\ell} \tilde{f}_{*}\right|^{2} \mid d v d v_{*} d \sigma \\
& \times \iiint b \theta^{\ell-2+\frac{3}{2}}|\tilde{g}|\left|h^{\prime}\right|^{2} d v d v_{*} d \sigma \\
= & B_{31} \times B_{32} .
\end{aligned}
$$

Then, if $\ell-2-\frac{3}{2}-2 s-1>-1$, that is, $\ell>2 s+\frac{7}{2}$, we have

$$
B_{31} \leq C\|\tilde{g}\|_{L^{1}}\|\tilde{f}\|_{L_{\ell}^{2}}^{2} .
$$


On the other hand, for $B_{32}$ we need to apply the singular change of variables $v_{*} \rightarrow v^{\prime}$. The Jacobian of this transform is, with $\mathbf{k}=\left(v-v_{*}\right) /\left|v-v_{*}\right|$,

$$
\left|\frac{\partial v_{*}}{\partial v^{\prime}}\right|=\frac{8}{|I-\mathbf{k} \otimes \sigma|}=\frac{8}{|1-\mathbf{k} \cdot \sigma|}=\frac{4}{\sin ^{2}(\theta / 2)} \leq 16 \theta^{-2}, \quad \theta \in[0, \pi / 2] .
$$

Notice that this gives rise to an additional singularity in the angle $\theta$ around 0 . Actually, the situation is even worse in the following sense. Recall that $\theta$ is no longer legitimate polar angle. In this case, the best choice of the pole is $\mathbf{k}^{\prime \prime}=\left(v^{\prime}-v\right) /\left|v^{\prime}-v\right|$ for which polar angle $\psi$ defined by $\cos \psi=\mathbf{k}^{\prime \prime} \cdot \sigma$ satisfies (cf. [1, Fig. 1])

$$
\psi=\frac{\pi-\theta}{2}, \quad d \sigma=\sin \psi d \psi d \phi, \quad \psi \in\left[\frac{\pi}{4}, \frac{\pi}{2}\right] .
$$

This measure does not cancel the singularity of $b(\cos \theta)$, unlike the case in the usual polar coordinates. Nevertheless, this singular change of variables yields

$$
\begin{aligned}
B_{32} & \lesssim \iiint b|\theta|^{\ell-2+\frac{3}{2}}|\tilde{g}|\left|h^{\prime}\right|^{2} d v d v_{*} d \sigma \\
& \lesssim \iint D_{1}\left(v, v^{\prime}\right)|\tilde{g}|\left|h^{\prime}\right|^{2} d v d v^{\prime},
\end{aligned}
$$

with

$$
D_{1}\left(v, v^{\prime}\right)=\int_{\mathbb{S}^{2}} \theta^{\ell-2+\frac{3}{2}-2} b(\cos \theta) d \sigma \lesssim \int_{\pi / 4}^{\pi / 2}\left(\frac{\pi}{2}-\psi\right)^{-2-2 s+\ell-2+\frac{3}{2}-2} d \psi<\infty,
$$

because of $\ell>\frac{7}{2}+2 s$. Therefore,

$$
B_{32} \lesssim\|\tilde{g}\|_{L^{1}}\|h\|_{L^{2}}^{2} \lesssim\|\tilde{g}\|_{L_{2}^{2}}\|h\|_{L^{2}}^{2},
$$

which concludes

$$
\left|B_{3}\right| \lesssim\|\tilde{f}\|_{L_{\ell}^{2}}\|\tilde{g}\|_{L_{2}^{2}}\|h\|_{L^{2}} .
$$

This together with (2.5) and (2.6) yield the desired estimate.

Before ending this section, we recall the upper and lower bound estimates. It follows from Proposition 2.9 of [5] that

Proposition 2.6. Let $0<s<1$ and $\gamma>\max \{-3,-2 s-3 / 2\}$. Then we have

$$
\begin{aligned}
& \left|(Q(f, g), h)_{L^{2}\left(\mathbb{R}_{v}^{3}\right)}\right| \\
& \quad \lesssim\left(\|f\|_{L_{(\gamma+2 s)^{+}}^{1}}\left(\mathbb{R}_{v}^{3}\right)\right. \\
& \left.\quad\|f\|_{L^{2}\left(\mathbb{R}_{v}^{3}\right)}\right)\|g\|_{H_{(\gamma+2 s)}^{2 s}}\left(\mathbb{R}_{v}^{3)}\right)\|h\|_{L^{2}\left(\mathbb{R}_{v}^{3}\right)} .
\end{aligned}
$$

Proposition 2.7. Let $0<s<1$ and $-3 / 2<\gamma \leq 0$. Then we have

$$
\begin{aligned}
(Q(f, h), h) & \leq-\frac{1}{2} \iiint B f_{*}\left(h-h^{\prime}\right)^{2} d v d v_{*} d \sigma \\
& +C\left(\|f\|_{L^{1}}+\|f\|_{L^{2}}\right)\|h\|_{L^{2}}^{2} .
\end{aligned}
$$

The above proposition is a direct consequence of the cancellation lemma [1]. 


\section{UNIFORM ESTIMATE}

In this section, we will obtain a uniform estimate for solutions in the given function space. We will start with the commutator estimate. For simplicity of notation, we denote $\phi_{a}=\phi(x-a, v)$. By using the fact that $\langle v\rangle \leq \mathcal{W}$, the following lemma on the commutator follows from Lemma 2.5 and Lemma 2.2.

Lemma 3.1. If $-3 / 2<\gamma \leq 0,0<s<1 / 2$ and $\ell \geq 5$, then

$$
\begin{gathered}
\left|\left(\left(\phi_{a} \mathcal{W}^{\ell} Q(f, g)-Q\left(f, \phi_{a} \mathcal{W}^{\ell} g\right)\right), h\right)_{L^{2}\left(\mathbb{R}^{6}\right)}\right| \\
\lesssim\|h\|\left[\operatorname { m i n } \left\{\left\|\phi_{a} \mathcal{W}^{5} f\right\|\left(\sup _{a^{\prime}}\left\|\left(1-\Delta_{x}\right) \phi_{a^{\prime}} \mathcal{W}^{\ell+2} g\right\|\right),\right.\right. \\
\left.\left(\sup _{a^{\prime}}\left\|\left(1-\Delta_{x}\right) \phi_{a^{\prime}} \mathcal{W}^{7} f\right\|\right)\left\|\phi_{a} \mathcal{W}^{\ell} g\right\|\right\} \\
+\min \left\{\left\|\phi_{a} \mathcal{W}^{\ell} f\right\|\left(\sup _{a^{\prime}}\left\|\left(1-\Delta_{x}\right) \phi_{a^{\prime}} \mathcal{W}^{4} g\right\|\right),\right. \\
\left.\left.\left(\sup _{a^{\prime}}\left\|\left(1-\Delta_{x}\right) \phi_{a^{\prime}} \mathcal{W}^{\ell+2} f\right\|\right)\left\|\phi_{a} \mathcal{W}^{2} g\right\|\right\}\right],
\end{gathered}
$$

where $\|\cdot\|=\|\cdot\|_{L^{2}\left(\mathbb{R}^{6}\right)}$.

Now by $\langle v\rangle^{2 s} \leq \mathcal{W}^{1-2 s}$, the following upper bound estimate follows from Proposition 2.6 and Lemma 2.2 .

Lemma 3.2. Let $0<s<1 / 2$ and $0 \geq \gamma>\max \{-3,-2 s-3 / 2\}$. Then

$$
\begin{aligned}
\mid\left(Q\left(f, \phi_{a} \mathcal{W}^{\ell} g\right), h\right)_{L^{2}\left(\mathbb{R}^{6}\right) \mid \lesssim} & \left(\sup _{a^{\prime}}\left\|\left(1-\Delta_{x}\right) \phi_{a^{\prime}} \mathcal{W}^{5} f\right\|\right) \\
& \left(\left\|\phi_{a} \mathcal{W}^{\ell+1} g\right\|+\left\|\phi_{a} \mathcal{W}^{\ell} \nabla_{v} g\right\|\right)\|h\|,
\end{aligned}
$$

and

$$
\begin{aligned}
&\left|\left(Q\left(f, \phi_{a} \mathcal{W}^{\ell} g\right), h\right)_{L^{2}\left(\mathbb{R}^{6}\right)}\right| \lesssim\left\|\phi_{a} \mathcal{W}^{5} f\right\| \\
&\left(\sup _{a^{\prime}}\left\|\left(1-\Delta_{x}\right) \phi_{a^{\prime}} \mathcal{W}^{\ell+3} g\right\|+\sup _{a^{\prime}}\left\|\left(1-\Delta_{x}\right) \phi_{a^{\prime}} \mathcal{W}^{\ell+2} \nabla_{v} g\right\|\right)\|h\| .
\end{aligned}
$$

Proof. Since $\langle v\rangle^{2 s} \leq \mathcal{W}^{1-2 s}$, it follows from Lemma 2.1 that we have

$$
\begin{aligned}
\|G\|_{H_{(\gamma+2 s)}^{2 s}}\left(\mathbb{R}_{v}^{3}\right) & \lesssim\left\|\mathcal{W}^{1-2 s}\left\langle D_{v}\right\rangle^{2 s} G\right\|_{L^{2}\left(\mathbb{R}_{v}^{3}\right)} \\
& \lesssim\|\mathcal{W} G\|_{L^{2}\left(\mathbb{R}_{v}^{3}\right)}+\left\|\nabla_{v} G\right\|_{L^{2}\left(\mathbb{R}_{v}^{3}\right)}
\end{aligned}
$$

Let $G=\phi_{a} \mathcal{W}^{\ell} g$, then the above estitmate together with Lemma 2.2 yield the first estimate in the lemma. The second estimate is a direct consequence of Lemma 2.3 .

By Proposition 2.7 together with Lemma 2.2, we have

Lemma 3.3. If $0<s<1,-3 / 2<\gamma \leq 0$ and $f \geq 0$, then we have

$$
\left(Q\left(f, \phi_{a} \mathcal{W}^{\ell} g\right), \phi_{a} \mathcal{W}^{\ell} g\right)_{L^{2}\left(\mathbb{R}^{6}\right)} \lesssim\left(\sup _{a^{\prime}}\left\|\left(1-\Delta_{x}\right) \phi_{a^{\prime}} \mathcal{W}^{4} f\right\|\right)\left\|\phi_{a} \mathcal{W}^{\ell} g\right\|^{2} .
$$


With the above preparation, we now perform the energy estimate for obtaining the uniform estimate on the solution. By differentiating the equation by $\partial_{x}^{\alpha} \partial_{v}^{\beta}$ with $|\alpha+\beta| \leq k$, where $k$ will be chosen later, and multiplying it by $\phi_{a} \mathcal{W}^{\ell-|\alpha+\beta|}$, we have

$$
\begin{aligned}
& \left(\partial_{t}+v \cdot \nabla_{x}\right) \phi_{a} \mathcal{W}^{\ell-|\alpha+\beta|} \partial_{\beta}^{\alpha} f-\phi_{a} \mathcal{W}^{\ell-|\alpha+\beta|} Q\left(f, \partial_{\beta}^{\alpha} f\right) \\
& =\left[v \cdot \nabla_{x}, \phi_{a} \mathcal{W}^{\ell-|\alpha+\beta|} \partial_{\beta}^{\alpha}\right] f \\
& \quad+\sum_{\left|\alpha^{\prime}+\beta^{\prime}\right| \neq 0} \frac{\alpha^{\prime} ! \beta^{\prime} ! \alpha^{\prime \prime} ! \beta^{\prime \prime} !}{\alpha ! \beta !} \phi_{a} \mathcal{W}^{\ell-|\alpha+\beta|} Q\left(\partial_{\beta^{\prime}}^{\alpha^{\prime}} f, \partial_{\beta^{\prime \prime}}^{\alpha^{\prime \prime}} f\right) .
\end{aligned}
$$

Therefore, if $f \geq 0$ then it follows from (3.4) and (3.1) that

$$
\begin{aligned}
& \left(\phi_{a} \mathcal{W}^{\ell-|\alpha+\beta|} Q\left(f, \partial_{\beta}^{\alpha} f\right), \phi_{a} \mathcal{W}^{\ell-|\alpha+\beta|} \partial_{\beta}^{\alpha} f\right)_{L^{2}\left(\mathbb{R}^{6}\right)} \\
& \lesssim\left(\sup _{a^{\prime}}\left\|\left(1-\Delta_{x}\right) \phi_{a^{\prime}} \mathcal{W}^{7} f\right\|\right)\left\|\phi_{a} \mathcal{W}^{\ell-|\alpha+\beta|} \partial_{\beta}^{\alpha} f\right\|^{2} \\
& +\left\|\phi_{a} \mathcal{W}^{\ell-|\alpha+\beta|} \partial_{\beta}^{\alpha} f\right\| \min \left\{\left\|\phi_{a} \mathcal{W}^{\ell-|\alpha+\beta|} f\right\|\left(\sup _{a^{\prime}}\left\|\left(1-\Delta_{x}\right) \phi_{a^{\prime}} \mathcal{W}^{4} \partial_{\beta}^{\alpha} f\right\|\right),\right. \\
& \left.\quad\left(\sup _{a^{\prime}}\left\|\left(1-\Delta_{x}\right) \phi_{a^{\prime}} \mathcal{W}^{\ell-|\alpha+\beta|+2} f\right\|\right)\left\|\phi_{a} \mathcal{W}^{2} \partial_{\beta}^{\alpha} f\right\|\right\} \\
& =A+B .
\end{aligned}
$$

If $|\alpha+\beta| \geq 4$ then

$$
\begin{aligned}
B & \lesssim\left\|\phi_{a} \mathcal{W}^{\ell-|\alpha+\beta|} \partial_{\beta}^{\alpha} f\right\|^{2}\left(\sup _{a^{\prime}}\left\|\left(1-\Delta_{x}\right) \phi_{a^{\prime}} \mathcal{W}^{\ell-|\alpha+\beta|+2} f\right\|\right) \\
& \lesssim\left\|\phi_{a} \mathcal{W}^{\ell-|\alpha+\beta|} \partial_{\beta}^{\alpha} f\right\|^{2}\left(\sum_{\left|\alpha^{\prime}\right| \leq 2} \sup _{a^{\prime}}\left\|\phi_{a^{\prime}} \mathcal{W}^{\ell-\left|\alpha^{\prime}\right|} \partial^{\alpha^{\prime}} f\right\|\right) .
\end{aligned}
$$

On the other hand, when $|\alpha+\beta| \leq 3$ then

$$
\begin{aligned}
B & \lesssim\left\|\phi_{a} \mathcal{W}^{\ell-|\alpha+\beta|} \partial_{\beta}^{\alpha} f\right\|\left\|\phi_{a} \mathcal{W}^{\ell} f\right\|\left(\sup _{a^{\prime}}\left\|\left(1-\Delta_{x}\right) \phi_{a^{\prime}} \mathcal{W}^{4} \partial_{\beta}^{\alpha} f\right\|\right) \\
& \lesssim\left\|\phi_{a} \mathcal{W}^{\ell-|\alpha+\beta|} \partial_{\beta}^{\alpha} f\right\|\left\|\phi_{a} \mathcal{W}^{\ell} f\right\|\left(\sum_{\left|\alpha^{\prime}+\beta\right| \leq 5} \sup _{a^{\prime}}\left\|\phi_{a^{\prime}} \mathcal{W}^{\ell-\left|\alpha^{\prime}+\beta\right|} \partial_{\beta}^{\alpha^{\prime}} f\right\|\right)
\end{aligned}
$$

when $\ell \geq 9$ is assumed, which is also enough to estimate $A$.

When $\left|\alpha^{\prime}+\beta^{\prime}\right| \neq 0$, by means of (3.2), (3.3) and (3.1) we have

$$
\begin{aligned}
& \left|\left(\phi_{a} \mathcal{W}^{\ell-|\alpha+\beta|} Q\left(\partial_{\beta^{\prime}}^{\alpha^{\prime}} f, \partial_{\beta^{\prime \prime}}^{\alpha^{\prime \prime}} f\right), \phi_{a} \mathcal{W}^{\ell-|\alpha+\beta|} \partial_{\beta}^{\alpha} f\right)_{L^{2}\left(\mathbb{R}^{6}\right)}\right| \\
& \lesssim\left\|\phi_{a} \mathcal{W}^{\ell-|\alpha+\beta|} \partial_{\beta}^{\alpha} f\right\|\left(D_{1}+D_{2}+D_{3}\right),
\end{aligned}
$$

where

$$
\begin{aligned}
& D_{1}=\min \left\{\left(\sup _{a^{\prime}}\left\|\left(1-\Delta_{x}\right) \phi_{a^{\prime}} \mathcal{W} \mathcal{W}^{5} \partial_{\beta^{\prime}}^{\alpha^{\prime}} f\right\|\right)\right. \\
& \times\left(\left\|\phi_{a} \mathcal{W}^{\ell-|\alpha+\beta|+1} \partial_{\beta^{\prime \prime}}^{\alpha^{\prime \prime}} f\right\|+\left\|\phi_{a} \mathcal{W}^{\ell-|\alpha+\beta|} \nabla_{v} \partial_{\beta^{\prime \prime}}^{\alpha^{\prime \prime}} f\right\|\right), \\
&\left\|\phi_{a} \mathcal{W}^{5} \partial_{\beta^{\prime}}^{\alpha^{\prime}} f\right\|\left(\sup _{a^{\prime}}\left\|\left(1-\Delta_{x}\right) \phi_{a^{\prime}} \mathcal{W}^{\ell-|\alpha+\beta|+3} \partial_{\beta^{\prime \prime}}^{\alpha^{\prime \prime}} f\right\|\right. \\
&\left.\left.\quad \sup _{a^{\prime}}\left\|\left(1-\Delta_{x}\right) \phi_{a^{\prime}} \mathcal{W}^{\ell-|\alpha+\beta|+2} \nabla_{v} \partial_{\beta^{\prime \prime}}^{\alpha^{\prime \prime}} f\right\|\right)\right\},
\end{aligned}
$$




$$
\begin{array}{r}
D_{2}=\min \left\{\left\|\phi_{a} \mathcal{W}^{5} \partial_{\beta^{\prime}}^{\alpha^{\prime}} f\right\|\left(\sup _{a^{\prime}}\left\|\left(1-\Delta_{x}\right) \phi_{a^{\prime}} \mathcal{W}^{\ell-|\alpha+\beta|+2} \partial_{\beta^{\prime \prime}}^{\alpha^{\prime \prime}} f\right\|\right),\right. \\
\left.\left(\sup _{a^{\prime}}\left\|\left(1-\Delta_{x}\right) \phi_{a^{\prime}} \mathcal{W}^{7} \partial_{\beta^{\prime}}^{\alpha^{\prime}} f\right\|\right)\left\|\phi_{a} \mathcal{W}^{\ell-|\alpha+\beta|} \partial_{\beta^{\prime \prime}}^{\alpha^{\prime \prime}} f\right\|\right\},
\end{array}
$$

and

$$
\begin{aligned}
D_{3}=\min \left\{\left\|\phi_{a} \mathcal{W}^{\ell-|\alpha+\beta|} \partial_{\beta^{\prime}}^{\alpha^{\prime}} f\right\|\left(\sup _{a^{\prime}}\left\|\left(1-\Delta_{x}\right) \phi_{a^{\prime}} \mathcal{W}^{4} \partial_{\beta^{\prime \prime}}^{\alpha^{\prime \prime}} f\right\|\right),\right. \\
\left.\left(\sup _{a^{\prime}}\left\|\left(1-\Delta_{x}\right) \phi_{a^{\prime}} \mathcal{W}^{\ell-|\alpha+\beta|+2} \partial_{\beta^{\prime}}^{\alpha^{\prime}} f\right\|\right)\left\|\phi_{a} \mathcal{W}^{2} \partial_{\beta^{\prime \prime}}^{\alpha^{\prime \prime}} f\right\|\right\} .
\end{aligned}
$$

If $1 \leq\left|\alpha^{\prime}+\beta^{\prime}\right| \leq k-2$ then $\left|\alpha^{\prime \prime}+\beta^{\prime \prime}\right| \leq|\alpha+\beta|-1$, from which we have

$$
\begin{aligned}
D_{1}+D_{2} \lesssim\left(\sup _{a^{\prime}}\right. & \left.\left\|\left(1-\Delta_{x}\right) \phi_{a^{\prime}} \mathcal{W}^{7} \partial_{\beta^{\prime}}^{\alpha^{\prime}} f\right\|\right) \\
& \times\left(\left\|\phi_{a} \mathcal{W}^{\ell-\left|\alpha^{\prime \prime}+\beta^{\prime \prime}\right|} \partial_{\beta^{\prime \prime}}^{\alpha^{\prime \prime}} f\right\|+\left\|\phi_{a} \mathcal{W}^{\ell-\left(\left|\alpha^{\prime \prime}+\beta^{\prime \prime}\right|+1\right)} \nabla_{v} \partial_{\beta^{\prime \prime}}^{\alpha^{\prime \prime}} f\right\|\right)
\end{aligned}
$$

provided that $\ell \geq k+7$. On the other hand, if $\left|\alpha^{\prime}+\beta^{\prime}\right| \geq k-1$ and $k \geq 6$, then we have

$$
\ell-|\alpha+\beta|+3=\ell-\left(\left|\alpha^{\prime \prime}+\beta^{\prime \prime}\right|+2\right)+5-\left|\alpha^{\prime}+\beta^{\prime}\right| \leq \ell-\left(\left|\alpha^{\prime \prime}+\beta^{\prime \prime}\right|+2\right)
$$

and therefore

$$
\begin{aligned}
& D_{1}+D_{2} \lesssim\left\|\phi_{a} \mathcal{W}^{5} \partial_{\beta^{\prime}}^{\alpha^{\prime}} f\right\|\left(\sup _{a^{\prime}}\left\|\left(1-\Delta_{x}\right) \phi_{a^{\prime}} \mathcal{W}^{\ell-|\alpha+\beta|+3} \partial_{\beta^{\prime \prime}}^{\alpha^{\prime \prime}} f\right\|\right. \\
& \\
&\left.\quad+\sup _{a^{\prime}}\left\|\left(1-\Delta_{x}\right) \phi_{a^{\prime}} \mathcal{W}^{\ell-|\alpha+\beta|+2} \nabla_{v} \partial_{\beta^{\prime \prime}}^{\alpha^{\prime \prime}} f\right\|\right) \\
& \lesssim\|f\|_{\mathcal{H}_{u l}^{k, \ell}}^{2} .
\end{aligned}
$$

It remains to estimate $D_{3}$. If $\left|\alpha^{\prime \prime}+\beta^{\prime \prime}\right| \leq k-2$, then

$$
D_{3} \lesssim\left\|\phi_{a} \mathcal{W}^{\ell-|\alpha+\beta|} \partial_{\beta^{\prime}}^{\alpha^{\prime}} f\right\|\left(\sup _{a^{\prime}}\left\|\left(1-\Delta_{x}\right) \phi_{a^{\prime}} \mathcal{W}^{4} \partial_{\beta^{\prime \prime}}^{\alpha^{\prime \prime}} f\right\|\right) \lesssim\|f\|_{\mathcal{H}_{u l}^{k, \ell}}^{2} .
$$

While, if $\left|\alpha^{\prime \prime}+\beta^{\prime \prime}\right| \geq k-1$, then it follows from $k \geq 6$ that

$$
\ell-|\alpha+\beta|+2 \leq \ell-\left(\left|\alpha^{\prime}+\beta^{\prime}\right|+2\right)-(k-1)+2<\ell-\left(\left|\alpha^{\prime}+\beta^{\prime}\right|+2\right),
$$

from which we have

$$
D_{3} \lesssim\left(\sup _{a^{\prime}}\left\|\left(1-\Delta_{x}\right) \phi_{a^{\prime}} \mathcal{W}^{\ell-|\alpha+\beta|+2} \partial_{\beta^{\prime}}^{\alpha^{\prime}} f\right\|\right)\left\|\phi_{a} \mathcal{W}^{2} \partial_{\beta^{\prime \prime}}^{\alpha^{\prime \prime}} f\right\| \lesssim\|f\|_{\mathcal{H}_{u l}^{k, \ell}}^{2} .
$$

Notice that

$$
\begin{aligned}
& \left\|\left[v \cdot \nabla_{x}, \phi_{a} \mathcal{W}^{\ell-|\alpha+\beta|} \partial_{\beta}^{\alpha}\right] f\right\| \\
& \lesssim \sum_{e_{j} \leq \beta}\left\|\phi_{a} \mathcal{W}^{\ell-|\alpha+\beta|} \partial_{\beta-e_{j}}^{\alpha+e_{j}} f\right\|+\left\|\phi_{a} \mathcal{W}^{\ell-|\alpha+\beta|} \partial_{\beta}^{\alpha} f\right\| \lesssim\|f\|_{\mathcal{H}_{u l}^{k, \ell}} .
\end{aligned}
$$

In summary, let $T>0$ and $f(t) \in C^{0}\left([0, T] ; \mathcal{H}_{u l}^{k, \ell}\left(\mathbb{R}^{6}\right)\right)$ with $k \geq 6$ and $\ell \geq k+7$. If we put

$$
\mathcal{E}(t)=\|f(t)\|_{\mathcal{H}_{u l}^{k, \ell}}^{2},
$$


then there exists a $C>0$ depending only on $s, \gamma, k, \ell$ and $K>0$ in the hypothesis of $b$ such that

$$
\mathcal{E}(t) \leq \mathcal{E}(0)+C \int_{0}^{t} \mathcal{E}(\tau)(1+\mathcal{E}(\tau)) d \tau, \quad t \in[0, T]
$$

To make the argument rigorous, in fact, we can choose $S(\tau) \in C_{0}^{\infty}(\mathbb{R})$ satisfies $S(\tau)=1$ for $|\tau| \leq 1$ and put $S_{N}\left(D_{x}\right)=S\left(\left|D_{x}\right|^{2} / N\right)$ for $N \in \mathbb{N}$, then we have

$$
\left(v \cdot \nabla_{x}\left(S_{N}\left(D_{x}\right) \phi_{a} \mathcal{W}^{\ell-|\alpha+\beta|} \partial_{\beta}^{\alpha} f\right), S_{N}\left(D_{x}\right) \phi_{a} \mathcal{W}^{\ell-|\alpha+\beta|} \partial_{\beta}^{\alpha} f\right)_{L^{2}\left(\mathbb{R}^{6}\right)}=0,
$$

and

$$
\left(F, S_{N}\left(D_{x}\right)^{2} G\right)_{L^{2}\left(\mathbb{R}^{6}\right)} \rightarrow(F, G)_{L^{2}\left(\mathbb{R}^{6}\right)},(N \rightarrow \infty), \quad F, G \in L^{2}\left(\mathbb{R}^{6}\right) .
$$

Therefore, multiplying $S_{N}\left(D_{x}\right)^{2} \phi_{a} \mathcal{W}^{\ell-|\alpha+\beta|} \partial_{\beta}^{\alpha} f$ by (3.5) and integrating with respect to $t, x$, by means of the limiting procedure $N \rightarrow \infty$, we have (3.6) in view of above estimations.

It follows from (3.6) that we have

$$
\mathcal{E}(t) \leq \frac{\mathcal{E}(0) e^{C t}}{1-\left(e^{C t}-1\right) \mathcal{E}(0)},
$$

by exactly the same calculation as the one after (4.3.11) of [3]. If we choose $T_{*}>0$ small enough such that

$$
T_{*}=\frac{1}{C} \log \left(1+\frac{3}{1+4\left\|f_{0}\right\|_{\mathcal{H}_{u l}^{k, \ell}\left(\mathbb{R}^{6}\right)}}\right)
$$

then we obtain a uniform estimate

$$
\|f(t)\|_{\mathcal{H}_{u l}^{k, \ell}\left(\mathbb{R}^{6}\right)} \leq 2\left\|f_{0}\right\|_{\mathcal{H}_{u l}^{k, \ell}\left(\mathbb{R}^{6}\right)} \text { for } t \in\left[0, T_{*}\right]
$$

\section{Cutoff Approximation}

To complete the proof of local existence, we still need to construct a sequence of approximate solutions.

As usual, we construct the approximate solutions by angular cutoff approximation. That is, for $0<\varepsilon \ll 1$, we approximate (cutoff) the cross section by

$$
b_{\epsilon}(\cos \theta)= \begin{cases}b(\cos \theta) & (\theta \geq 2 \epsilon), \\ 0 & (\theta<2 \epsilon) .\end{cases}
$$

Theorem 4.1 (Cutoff case). Assume that $-3 / 2<\gamma \leq 0$ and replace the angular factor of the cross section $b$ by $b_{\varepsilon}$. If the initial data $f_{0}$ is non-negative and belongs to $\mathcal{H}_{u l}^{k, \ell}\left(\mathbb{R}^{6}\right)$ for $k \geq 5, \ell \geq k+7$, then, there exists a $T_{\varepsilon}>0$ such that the Cauchy problem (1.1) admits a non-negative unique solution $f^{\varepsilon}(t, x, v)$ in the function space $C^{0}\left(\left[0, T_{\varepsilon}\right] ; \mathcal{H}_{u l}^{k, \ell}\left(\mathbb{R}^{6}\right)\right)$.

Remark 4.2. In the cutoff case, the order of derivative $k$ can be taken not less than 5 instead of 6 for the non-cutoff case in our analysis.

To prove this theorem, define a sequence of successive approximate solutions $\left\{f^{n}\right\}_{n \in \mathbb{N}}$ by

$$
\left\{\begin{array}{l}
f^{0}=f_{0} \\
\partial_{t} f^{n+1}+v \cdot \nabla_{x} f^{n+1}=Q_{\varepsilon}^{+}\left(f^{n}, f^{n}\right)-Q_{\varepsilon}^{-}\left(f^{n}, f^{n+1}\right), \\
\left.f^{n+1}\right|_{t=0}=f_{0} .
\end{array}\right.
$$


Here

$$
\begin{aligned}
Q_{\varepsilon}^{+}(g, h) & =\iint_{\mathbb{R}_{v_{*}}^{3} \times \mathbb{S}_{\sigma}^{2}} B_{\varepsilon}\left(v-v_{*}, \sigma\right) g_{*}^{\prime} h^{\prime} d v_{*} d \sigma, \\
Q_{\varepsilon}^{-}(g, h) & =h L_{\varepsilon}(g), \\
L_{\varepsilon}(g) & =\iint_{\mathbb{R}_{v_{*}}^{3} \times \mathbb{S}_{\sigma}^{2}} B_{\varepsilon}\left(v-v_{*}, \sigma\right) g_{*} d v_{*} d \sigma .
\end{aligned}
$$

Notice that

$$
\begin{aligned}
& \left|\partial_{\beta}^{\alpha} L_{\varepsilon}(f)(t, x, v)\right|=\left|L_{\varepsilon}\left(\partial_{\beta}^{\alpha} f\right)(t, x, v)\right| \\
& \leq C \int\left(\left|v-v_{*}\right|^{\gamma} \mathbf{1}_{\left|v-v_{*}\right| \leq 1}+\mathbf{1}_{\left|v-v_{*}\right| \geq 1}\right)\left|\left(\partial_{\beta}^{\alpha} f\right)\left(t, x, v_{*}\right)\right| d v_{*} \\
& \leq C\left\{\left(\int\left|v-v_{*}\right|^{2 \gamma} \mathbf{1}_{\left|v-v_{*}\right| \leq 1} d v_{*}\right)^{1 / 2}\left\|\partial_{\beta}^{\alpha} f(t, x, \cdot)\right\|_{L_{v}^{2}}+\left\|\partial_{\beta}^{\alpha} f(t, x, \cdot)\right\|_{L_{v}^{1}}\right\} \\
& \leq C\left\|\partial_{\beta}^{\alpha} f(t, x, \cdot)\right\|_{L_{3 / 2+\varepsilon^{\prime}}^{2}\left(\mathbb{R}_{v}^{3}\right)}, \quad t \in\left[0, T_{0}\right],
\end{aligned}
$$

for a constant $C>0$ depending on $\varepsilon$. Here we have used $\gamma>-3 / 2$. Putting

$$
V^{n}(t, s, x, v)=\int_{s}^{t} L_{\varepsilon}\left(f^{n}\right)(\tau, x-(t-\tau) v, v) d \tau \quad\left(\geq 0 \text { if } f_{n} \geq 0\right),
$$

in the following, we will consider the solution in the mild form

$$
\begin{aligned}
& f^{n+1}(t, x, v)=e^{-V^{n}(t, 0, x, v)} f_{0}(x-t v, v) \\
& \quad+\int_{0}^{t} e^{-V^{n}(t, s, x, v)} Q_{\varepsilon}^{+}\left(f^{n}, f^{n}\right)(s, x-(t-s) v, v) d s .
\end{aligned}
$$

Note that

$$
\begin{aligned}
& \partial_{\beta}^{\alpha} V^{n}(t, s, x, v) \\
& \quad=\sum_{\beta^{\prime}+\beta^{\prime \prime}=\beta} \int_{s}^{t} \frac{(\tau-t)^{\left|\beta^{\prime \prime}\right|} \beta !}{\beta^{\prime} ! \beta^{\prime \prime} !} L_{\varepsilon}\left(\partial_{\beta^{\prime}}^{\alpha+\beta^{\prime \prime}} f^{n}\right)(\tau, x-(t-\tau) v, v) d \tau,
\end{aligned}
$$

and

$$
\partial_{\beta}^{\alpha} Q_{\varepsilon}^{+}(g, h)(x, v)=\sum_{\substack{\alpha^{\prime}+\alpha^{\prime \prime}=\alpha \\ \beta^{\prime}+\beta^{\prime \prime}=\beta}} \frac{\alpha ! \beta !}{\alpha^{\prime} ! \beta^{\prime} ! \alpha^{\prime \prime} ! \beta^{\prime \prime} !} Q_{\varepsilon}^{+}\left(\partial_{\beta^{\prime}}^{\alpha^{\prime}} g, \partial_{\beta^{\prime \prime}}^{\alpha^{\prime \prime}} h\right)(x, v)
$$

Furthermore, notice that

$$
\begin{aligned}
& \partial_{\beta}^{\alpha} Q_{\varepsilon}^{+}(g, h)(x-(t-\tau) v, v) \\
& =\sum_{\tilde{\beta}^{\prime}+\tilde{\beta}^{\prime \prime}=\beta} \frac{(\tau-t)^{\left|\tilde{\beta}^{\prime \prime}\right|} \beta !}{\tilde{\beta}^{\prime} ! \tilde{\beta}^{\prime \prime} !}\left(\partial_{\tilde{\beta}^{\prime}}^{\alpha+\tilde{\beta}^{\prime \prime}} Q_{\varepsilon}^{+}(g, h)\right)(x-(t-\tau) v, v) \\
& =\sum_{\tilde{\beta}^{\prime}+\tilde{\beta}^{\prime \prime}=\beta} \frac{(\tau-t)^{\left|\tilde{\beta}^{\prime \prime}\right|} \beta !}{\tilde{\beta}^{\prime} ! \tilde{\beta}^{\prime \prime} !} \\
& \quad \times\left(\sum_{\substack{\alpha^{\prime}+\alpha^{\prime \prime}=\alpha+\tilde{\beta}^{\prime \prime} \\
\beta^{\prime}+\beta^{\prime \prime}=\tilde{\beta}^{\prime}}} \frac{\alpha ! \beta !}{\alpha^{\prime} ! \beta^{\prime} ! \alpha^{\prime \prime} ! \beta^{\prime \prime !} !} Q_{\varepsilon}^{+}\left(\partial_{\beta^{\prime}}^{\alpha^{\prime}} g, \partial_{\beta^{\prime \prime}}^{\alpha^{\prime \prime}} h\right)(x-(t-\tau), v)\right) .
\end{aligned}
$$


Then, by applying Lemma 2.2 we have

$$
\begin{aligned}
& \left\|V^{n}(t, s, \cdot, \cdot)\right\|_{L^{\infty}} \lesssim(t-s) \sup _{s \leq \tau \leq t}\left\|f^{n}(\tau, \cdot, \cdot)\right\|_{L^{\infty}\left(\mathbb{R}_{x}^{3}, L_{3 / 2+\varepsilon^{\prime}}^{2}\left(\mathbb{R}_{v}^{3}\right)\right)} \\
& \lesssim(t-s) \sup _{s \leq \tau \leq t}\left(\sup _{a \in \mathbb{R}_{x}^{3}}\left\|\left(1-\Delta_{x}\right) \phi_{a} \mathcal{W}^{4} f^{n}(\tau, x, \cdot)\right\|_{L^{2}\left(\mathbb{R}^{6}\right)}\right) .
\end{aligned}
$$

Furthermore

$$
\begin{aligned}
& \left\|\partial_{\beta}^{\alpha} V^{n}(t, s, \cdot, \cdot)\right\|_{L^{\infty}} \\
& \lesssim(t-s) \sup _{s \leq \tau \leq t} \sum_{|q|=|\alpha+\beta|} \sup _{s \leq \tau \leq t}\left(\sup _{a \in \mathbb{R}_{x}^{3}}\left\|\left(1-\Delta_{x}\right) \phi_{a} \partial^{q} \mathcal{W}^{4} f^{n}(\tau, x, \cdot)\right\|_{L^{2}\left(\mathbb{R}^{6}\right)}\right) .
\end{aligned}
$$

Here $\partial^{q}=\partial_{x}^{\alpha^{\prime}} \partial_{v}^{\beta^{\prime}}$ with $\alpha^{\prime}, \beta^{\prime}$ satisfying $\left|\alpha^{\prime}+\beta^{\prime}\right|=|q|$.

In order to obtain a variant of (4.5) which includes the factor $\phi_{a}$, we recall the property $\phi_{a}(x, v) \leq\left\langle v_{*}\right\rangle^{2} \phi_{a}\left(x, v_{*}\right)$ and prepare a simple lemma about the translation invariance of $\phi_{a}$ in finite time.

Lemma 4.3. For $|t| \leq T$, then we have

$$
\phi_{a}(x, v) \leq \max \left\{2,4 T^{2}\right\} \phi_{a}(x-t v, v)=\max \left\{2,4 T^{2}\right\} \phi_{a+t v}(x, v) .
$$

Proof. This follows from

$$
\langle v\rangle^{2}+|x-t v-a|^{2} \leq\langle v\rangle^{2}+2|x-a|^{2}+2 t^{2}|v|^{2} \leq \max \left\{2,4 T^{2}\right\} \phi_{a}(x, v)^{-1} .
$$

From now on, to be concrete, we take $T<\frac{1}{2}$. It follows from (4.4) and the above lemma that

$$
\begin{aligned}
\left|\phi_{a}(x, v) \partial_{\beta}^{\alpha} V^{n}(t, s, x, v)\right|^{2} & \\
& \lesssim \sum_{|q|=|\alpha+\beta|} \int_{s}^{t}\left|\phi_{a+(t-\tau) v}(x, v) L_{\varepsilon}\left(\partial^{q} f^{n}\right)(\tau, x-(t-\tau) v, v)\right|^{2} d \tau \\
& \lesssim \sum_{|q|=|\alpha+\beta|} \int_{s}^{t}\left\|\left(\phi_{a} \mathcal{W}^{4} \partial^{q} f^{n}\right)(\tau, x-(t-\tau) v, \cdot)\right\|_{L^{2}\left(\mathbb{R}^{3}\right)}^{2} d \tau,
\end{aligned}
$$

where, in the second inequality, we have used a variant of (4.2), namely,

$$
\begin{aligned}
& \left|\phi_{a}(x, v) L_{\varepsilon}\left(\partial^{q} f\right)(t, x, v)\right| \\
& \lesssim \int\left(\left|v-v_{*}\right|^{\gamma} \mathbf{1}_{\left|v-v_{*}\right| \leq 1}+\mathbf{1}_{\left|v-v_{*}\right| \geq 1}\right)\left|\phi_{a}\left(x, v_{*}\right)\left\langle v_{*}\right\rangle^{2}\left(\partial^{q} f\right)\left(t, x, v_{*}\right)\right| d v_{*} \\
& \lesssim\left(\int\left|\left(\phi_{a} \mathcal{W}^{4} \partial^{q} f^{n}\right)\left(t, x, v_{*}\right)\right|^{2} d v_{*}\right)^{1 / 2},
\end{aligned}
$$

with $x=x-(t-\tau) v$.

Moreover, observe that

$$
\left\|\phi_{a} \mathcal{W}^{\ell} e^{-V^{n}} f_{0}(x-t v, v)\right\|_{L^{2}\left(\mathbb{R}^{6}\right)} \leq 2 \sup _{a^{\prime}}\left\|\phi_{a^{\prime}} \mathcal{W}^{\ell} f_{0}\right\|_{L^{2}\left(\mathbb{R}^{6}\right)}
$$

Proposition 4.4. Assume that $-3 / 2<\gamma \leq 0$. Let $\varepsilon>0$ and $D_{0}>0$. Then there exists a $T_{\varepsilon}^{\prime}>0$ such that for any $f_{0}(x, v)$ satisfying

$$
f_{0} \geq 0, \quad\left\|f_{0}\right\|_{\mathcal{H}_{u l}^{k, \ell}}^{2} \leq D_{0}^{2}
$$


with $k \geq 5$ and $\ell \geq k+7$, we have for any $n \geq 0$

$$
f^{n} \geq 0, \quad\left\|f^{n}(t)\right\|_{\mathcal{H}_{u l}^{k, \ell}}^{2} \leq 6 D_{0}^{2} \text { for } t \in\left[0, T_{\varepsilon}\right] .
$$

Proof. In view of (4.3), for $q \in \mathbb{Z}_{+}^{6}$ with $|q| \leq k$ we consider

$$
\begin{aligned}
& \partial^{q}\left(e^{-V^{n}(t, s, x, v)} Q_{\varepsilon}^{+}\left(f^{n}, f^{n}\right)(s, x-(t-s) v, v)\right) \\
& =\sum_{p+q^{\prime}=q} \frac{q !}{p ! q^{\prime} !} \partial^{p}\left(e^{-V^{n}(t, s, x, v)}\right) \partial^{q^{\prime}}\left(Q_{\varepsilon}^{+}\left(f^{n}, f^{n}\right)(s, x-(t-s) v, v)\right) .
\end{aligned}
$$

The Faà di Bruno formula gives

$$
\partial^{p}\left(e^{-V^{n}}\right)=e^{-V^{n}} \sum_{j \leq|p|} \sum_{p_{1}+\cdots+p_{j}=p} C_{p_{1}, \ldots, p_{j}} \partial^{p_{1}}\left(-V^{n}\right) \cdots \partial^{p_{j}}\left(-V^{n}\right) .
$$

Note that

$$
\begin{aligned}
& \left|\partial^{p}\left(e^{-V^{n}(t, s, x, v)}\right) \partial^{q^{\prime}}\left(Q_{\varepsilon}^{+}\left(f^{n}, f^{n}\right)(s, x-(t-s) v, v)\right)\right|^{2} \\
& \lesssim \sum_{j \leq|p|} \sum_{p_{1}+\cdots+p_{j}=p}\left|\partial^{p_{1}}\left(V^{n}\right) \cdots \partial^{p_{j}}\left(V^{n}\right) \partial^{q^{\prime}}\left(Q_{\varepsilon}^{+}\left(f^{n}, f^{n}\right)(s, x-(t-s) v, v)\right)\right|^{2}
\end{aligned}
$$

because $V^{n} \geq 0$. First we consider the case $|p| \geq k-1$ and the term with $\left|p_{1}\right| \geq k-1$. Since $\left|p_{2}\right|, \ldots,\left|p_{j}\right| \leq k-2$, it follows from (4.5) and (4.6) that if we denote the integration of its corresponding term with product of weight $\phi_{a}$ by $J\left(p, q^{\prime}\right)$, then we have

$$
\begin{aligned}
& J\left(p, q^{\prime}\right) \leq\left(2 D_{0}\right)^{2 j} \iint_{\mathbb{R}_{x}^{3} \times \mathbb{R}_{v}^{3}} \mid \phi_{a} \mathcal{W}^{\ell-|q|} \partial^{p_{1}}\left(V^{n}\right) \cdots \partial^{p_{j}}\left(V^{n}\right) \\
& \times\left.\partial^{q^{\prime}}\left(Q_{\varepsilon}^{+}\left(f^{n}, f^{n}\right)(s, x-(t-s) v, v)\right)\right|^{2} d x d v \\
& \lesssim\left(2 D_{0}\right)^{2(j-1)} \iint_{\mathbb{R}_{x}^{3} \times \mathbb{R}_{v}^{3}}\left|\left(\phi_{a} \partial^{p_{1}} V^{n}\right)(t, s, x, v)\right|^{2} \\
& \quad \times\left|\mathcal{W}^{\ell-|q|} \partial^{q^{\prime}}\left(Q_{\varepsilon}^{+}\left(f^{n}, f^{n}\right)(s, x-(t-s) v, v)\right)\right|^{2} d x d v \\
& \lesssim\left(2 D_{0}\right)^{2(j-1)} \sum_{\left|\tilde{p}_{1}\right|=\left|p_{1}\right|} \int_{s}^{t} \iint_{\mathbb{R}_{x}^{3} \times \mathbb{R}_{v}^{3}}\left\|\left(\phi_{a} \mathcal{W}^{4} \partial^{\tilde{p}_{1}} f^{n}\right)(\tau, x, \cdot)\right\|_{L^{2}\left(\mathbb{R}^{3}\right)}^{2} \\
& \quad \times\left|\mathcal{W}^{\ell-|q|} \partial^{q^{\prime}}\left(Q_{\varepsilon}^{+}\left(f^{n}, f^{n}\right)(s, x-(\tau-s) v, v)\right)\right|^{2} d x d v d \tau \\
& \lesssim\left(2 D_{0}\right)^{2(j-1)} \sum_{\left|\tilde{p}_{1}\right|=\left|p_{1}\right|} \int_{s}^{t} \int_{\mathbb{R}_{x}^{3}}\left\|\left(\phi_{a} \mathcal{W}^{4} \partial^{\tilde{p}_{1}} f^{n}\right)(\tau, x, \cdot)\right\|_{L^{2}\left(\mathbb{R}^{3}\right)}^{2} d x d \tau \\
& \quad \times\left\|\mathcal{W}^{\ell-|q|} \partial^{q^{\prime}}\left(Q_{\varepsilon}^{+}\left(f^{n}, f^{n}\right)\right)\right\|_{L^{\infty}\left(\mathbb{R}_{x}^{3}, L^{2}\left(\mathbb{R}_{v}^{3}\right)\right)}^{2} \\
& \lesssim(t-s)\left(2 D_{0}\right)^{2 j+4},
\end{aligned}
$$

where in the third inequality, we have used the translation $x-(t-\tau) v \rightarrow x$; and the fifth inequality follows from (5.4), in view of $\left|q^{\prime}\right| \leq 1(|p| \geq k-1)$. 
The other cases are now easy. For example, if $|p| \leq k-2$, then it follows from (4.5) that

$$
\begin{aligned}
& J\left(p, q^{\prime}\right) \leq\left(2 D_{0}\right)^{2 j} \iint_{\mathbb{R}_{x}^{3} \times \mathbb{R}_{v}^{3}}\left|\phi_{a} \mathcal{W}^{\ell-|q|} \partial^{q^{\prime}}\left(Q_{\varepsilon}^{+}\left(f^{n}, f^{n}\right)(s, x-(t-s) v, v)\right)\right|^{2} d x d v \\
& \lesssim\left(2 D_{0}\right)^{2 j} \sum_{q_{1}+q_{2}=q^{\prime}} \iint_{\mathbb{R}_{x}^{3} \times \mathbb{R}_{v}^{3}}\left|\phi_{a-(t-s) v} \mathcal{W}^{\ell-|q|}\left(Q_{\varepsilon}^{+}\left(\partial^{q_{1}} f^{n}, \partial^{q_{2}} f^{n}\right)(s, x, v)\right)\right|^{2} d x d v \\
& \leq C_{\varepsilon} D_{0}^{2 j+4}
\end{aligned}
$$

by means of (5.3) and Lemma 4.3 .

Finally, if $g^{n+1}(t, x, v)$ denotes the second term of the right hand side of (4.3) then

$$
\left\|g^{n+1}(t)\right\|_{\mathcal{H}_{u l}^{k, \ell}}^{2} \leq C_{\varepsilon} t\left(D_{0}^{4}+D_{0}^{2 k+4}\right) .
$$

It is not difficult to see

$$
\left\|e^{-V^{n}(t, 0, x, v)} f_{0}(x-t v, v)\right\|_{\mathcal{H}_{u l}^{k, \ell}}^{2} \leq 4 D_{0}^{2}+C_{\varepsilon} t\left(D_{0}^{2}+D_{0}^{2 k+4}\right) .
$$

Hence, it is straightforward to check that for any given $k$ and $D_{0}$, there exists a time $T_{\varepsilon}^{\prime}>0$ such that (4.7) holds. And this completes the proof of the proposition.

Proposition 4.5. Assume that $-3 / 2<\gamma \leq 0$. Let $\varepsilon>0$ and $D_{0}>0$. Assume that

$$
f_{0} \geq 0, \quad\left\|f_{0}\right\|_{\mathcal{H}_{u l}^{k, \ell}}^{2} \leq D_{0}^{2}
$$

with $k \geq 5$ and $\ell \geq k+7$. Then for any $\delta>0$, there exists another $T_{\varepsilon}^{\prime \prime}>0$ such that

$$
\sup _{t \in\left[0, T_{\varepsilon}^{\prime \prime}\right]}\left\|f^{n+1}(t)-f^{n}(t)\right\|_{\mathcal{H}_{u l}^{k, \ell}} \leq(1-\delta) \sup _{t \in\left[0, T_{\varepsilon}^{\prime \prime}\right]}\left\|f^{n}(t)-f^{n-1}(t)\right\|_{\mathcal{H}_{u l}^{k, \ell}} .
$$

Proof. If we put $w^{n}=f^{n+1}-f^{n}$, then

$$
\begin{aligned}
& \partial_{t} w^{n}+v \cdot \nabla_{x} w^{n}+L_{\varepsilon}\left(f^{n}\right) w^{n}= \\
& \quad-L_{\varepsilon}\left(w^{n-1}\right) f^{n}+Q_{\varepsilon}^{+}\left(w^{n-1}, f^{n}\right)+Q_{\varepsilon}^{+}\left(f^{n-1}, w^{n-1}\right):=R_{\varepsilon}^{n}(t, x, v),
\end{aligned}
$$

so that we have

$$
w^{n}(t, x, v)=\int_{0}^{t} e^{-V^{n}(t, s, x, v)} R_{\varepsilon}^{n}(s, x-(t-s) v, v) d s .
$$

Therefore, by the almost same argument used in the proof of Lemma 4.4, we have

$$
\left\|w^{n}(t)\right\|_{\mathcal{H}_{u l}^{k, \ell}}^{2} \leq C_{\varepsilon} t\left(D_{0}^{2}+D_{0}^{2 k+2}\right) \sup _{0 \leq \tau \leq t}\left\|w^{n-1}(\tau)\right\|_{\mathcal{H}_{u l}^{k, \ell}}^{2} .
$$

If we put $T_{\varepsilon}=\min \left\{T_{\varepsilon}^{\prime}, T_{\varepsilon}^{\prime \prime}\right\}$. then Theorem 4.1 is a direct consequence of Propositions 4.4 and 4.5 .

The proof of Theorem 1.1 can be completed in the almost same way as in the proof of Theorem 4.11 of [3] and the subsequent paragraph there, taking into account the uniform estimate (3.7) and Theorem 4.1. 


\section{Estimate FOR $Q_{\varepsilon}^{+}$}

In this last section, we will complete the estimate on $Q_{\varepsilon}^{+}$that has been used in the previous section. We start with an almost obvious lemma.

Lemma 5.1. Let $0 \geq \gamma>-3 / 2$. Then there exists a $C_{\varepsilon}>0$ such that

$$
\left\|Q_{\varepsilon}^{+}(f, g)\right\|_{L^{2}\left(\mathbb{R}_{v}^{3}\right)} \leq C_{\varepsilon}\|f\|_{L_{2}^{2}\left(\mathbb{R}_{v}^{3}\right)}\|g\|_{L^{2}\left(\mathbb{R}_{v}^{3}\right)} .
$$

Proof. It follows from the Cauchy-Schwarz inequality and the change of variables $\left(v, v_{*}, \sigma\right) \rightarrow\left(v^{\prime}, v_{*}^{\prime}, \mathbf{k}\right),\left(\mathbf{k}=\left(v-v_{*}\right) /\left|v-v_{*}\right|\right)$ that

$$
\begin{aligned}
\left|\left(Q_{\varepsilon}^{+}(f, g), h\right)_{L^{2}\left(\mathbb{R}_{v}^{3}\right)}\right| \leq & \left(\iiint_{\mathbb{R}_{v}^{3} \times \mathbb{R}_{v_{*}}^{3} \times \mathbb{S}_{\sigma}^{2}} B_{\varepsilon}\left(v-v_{*}, \sigma\right)\left|f_{*}\right||g|^{2} d v d v_{*} d \sigma\right)^{2} \\
& \times\left(\iiint_{\mathbb{R}_{v}^{3} \times \mathbb{R}_{v_{*}}^{3} \times \mathbb{S}_{\sigma}^{2}} B_{\varepsilon}\left(v-v_{*}, \sigma\right)\left|f_{*}\right|\left|h^{\prime}\right|^{2} d v d v_{*} d \sigma\right)^{2} \\
= & A_{1} \times A_{2} .
\end{aligned}
$$

By means of the regular change of variables $v \rightarrow v^{\prime}$, we have

$$
\begin{aligned}
A_{2}^{2} & \leq C_{\varepsilon} \iint\left|v^{\prime}-v_{*}\right|^{\gamma}\left|f_{*}\right|\left|h^{\prime}\right|^{2} d v^{\prime} d v_{*} \\
& \leq C_{\varepsilon} \int\left|h^{\prime}\right|^{2}\left(\int_{\left|v^{\prime}-v_{*}\right| \leq 1}\left|v^{\prime}-v_{*}\right|^{\gamma}\left|f_{*}\right| d v_{*}+\int_{\left|v^{\prime}-v_{*}\right|>1}\left|f_{*}\right| d v_{*}\right) d v^{\prime} \\
& \leq C_{\varepsilon}^{\prime}\|h\|_{L^{2}}\left(\|f\|_{L^{2}}+\|f\|_{L^{1}}\right) .
\end{aligned}
$$

Similarly, we get $A_{1}^{2} \leq C_{\varepsilon}\|g\|_{L^{2}}\left(\|f\|_{L^{2}}+\|f\|_{L^{1}}\right)$ so that the proof of the lemma is completed.

Since it follows that

$$
\phi_{a} \mathcal{W}^{\ell} Q_{\varepsilon}^{+}(f, g)-Q_{\varepsilon}^{+}\left(f, \phi_{a} \mathcal{W}^{\ell}\right)=\phi_{a} \mathcal{W}^{\ell} Q_{\varepsilon}(f, g)-Q_{\varepsilon}\left(f, \phi_{a} \mathcal{W}^{\ell}\right),
$$

similar to Lemma 2.5, we have

Lemma 5.2. If $-3 / 2<\gamma \leq 0,0<s<1 / 2$ and $\ell \geq 5$, then there exists a $C>0$ independent of $\varepsilon>0$ such that

$$
\begin{aligned}
& \left\|\left(\phi_{a} \mathcal{W}^{\ell} Q_{\varepsilon}^{+}(f, g)-Q_{\varepsilon}^{+}\left(f, \phi_{a} \mathcal{W}^{\ell} g\right)\right)\right\|_{L^{2}\left(\mathbb{R}_{v}^{3}\right)} \\
& \leq C \min \left\{\left\|\phi_{a} \mathcal{W}^{5} f\right\|_{L^{2}\left(\mathbb{R}_{v}^{3}\right)}\left\|\mathcal{W}^{\ell} g\right\|_{L^{2}\left(\mathbb{R}_{v}^{3}\right)},\left\|\mathcal{W}^{5} f\right\|_{L^{2}\left(\mathbb{R}_{v}^{3}\right)}\left\|\phi_{a} \mathcal{W}^{\ell} g\right\|_{L^{2}\left(\mathbb{R}_{v}^{3}\right)}\right\} \\
& +\min \left\{\left\|\phi_{a} \mathcal{W}^{\ell} f\right\|_{L^{2}\left(\mathbb{R}_{v}^{3}\right)},\left\|\mathcal{W}^{2} g\right\|_{L^{2}\left(\mathbb{R}_{v}^{3}\right)}\left\|\mathcal{W}^{\ell} f\right\|_{L^{2}\left(\mathbb{R}_{v}^{3}\right)}\left\|\phi_{a} \mathcal{W}^{2} g\right\|_{L^{2}\left(\mathbb{R}_{v}^{3}\right)}\right\},
\end{aligned}
$$

by regarding $x, a$ in $\phi_{a}$ as parameters.

Notice that

$$
\left\|\mathcal{W}^{2} f\right\|_{L^{2}\left(\mathbb{R}_{v}^{3}\right)}\left\|\phi_{a} \mathcal{W}^{\ell} g\right\|_{L^{2}\left(\mathbb{R}_{v}^{3}\right)} \leq\left\|\phi_{a} \mathcal{W}^{4} f\right\|_{L^{2}\left(\mathbb{R}_{v}^{3}\right)}\left\|\mathcal{W}^{\ell} g\right\|_{L^{2}\left(\mathbb{R}_{v}^{3}\right)}
$$

because of $\phi_{a}(x, v) \leq\left\langle v_{*}\right\rangle^{2} \phi\left(x, v_{*}\right)$. Therefore, it follows from Lemma 5.1 and Lemma 5.2 to have 
Corollary 5.3. If $-3 / 2<\gamma \leq 0,0<s<1 / 2$ and $\ell \geq 5$, then there exists a $C_{\varepsilon}>0$ such that

$$
\begin{aligned}
& \left\|\phi_{a} \mathcal{W}^{\ell} Q_{\varepsilon}^{+}(f, g)\right\|_{L^{2}\left(\mathbb{R}_{v}^{3}\right)} \\
& \leq C_{\varepsilon} \min \left\{\left\|\phi_{a} \mathcal{W}^{5} f\right\|_{L^{2}\left(\mathbb{R}_{v}^{3}\right)}\left\|\mathcal{W}^{\ell} g\right\|_{L^{2}\left(\mathbb{R}_{v}^{3}\right)},\left\|\mathcal{W}^{5} f\right\|_{L^{2}\left(\mathbb{R}_{v}^{3}\right)}\left\|\phi_{a} \mathcal{W}^{\ell} g\right\|_{L^{2}\left(\mathbb{R}_{v}^{3}\right)}\right\} \\
& +\min \left\{\left\|\phi_{a} \mathcal{W}^{\ell} f\right\|_{L^{2}\left(\mathbb{R}_{v}^{3}\right)}\left\|\mathcal{W}^{2} g\right\|_{L^{2}\left(\mathbb{R}_{v}^{3}\right)},\left\|\mathcal{W}^{\ell} f\right\|_{L^{2}\left(\mathbb{R}_{v}^{3}\right)}\left\|\phi_{a} \mathcal{W}^{2} g\right\|_{L^{2}\left(\mathbb{R}_{v}^{3}\right)}\right\}
\end{aligned}
$$

by regarding $x, a$ in $\phi_{a}$ as parameters.

It follows from this corollary and Lemma 2.2 that if $\left|\alpha^{\prime}+\alpha^{\prime \prime}\right|+\left|\beta^{\prime}+\beta^{\prime \prime}\right| \leq|\alpha+\beta|$, then

$$
\left\|\phi_{a} \mathcal{W}^{\ell-|\alpha+\beta|} Q_{\varepsilon}^{+}\left(\partial_{\beta^{\prime}}^{\alpha^{\prime}} f, \partial_{\beta^{\prime \prime}}^{\alpha^{\prime \prime}} g\right)\right\|_{L^{2}\left(\mathbb{R}^{6}\right)} \lesssim E_{1}+E_{2}
$$

where

$$
\begin{array}{r}
E_{1}=\min \left\{\left\|\phi_{a} \mathcal{W}^{5} \partial_{\beta^{\prime}}^{\alpha^{\prime}} f\right\|\left(\sup _{a^{\prime}}\left\|\left(1-\Delta_{x}\right) \phi_{a^{\prime}} \mathcal{W}^{\ell-|\alpha+\beta|+2} \partial_{\beta^{\prime \prime}}^{\alpha^{\prime \prime}} f\right\|\right),\right. \\
\left.\left(\sup _{a^{\prime}}\left\|\left(1-\Delta_{x}\right) \phi_{a^{\prime}} \mathcal{W}^{7} \partial_{\beta^{\prime}}^{\alpha^{\prime}} f\right\|\right)\left\|\phi_{a} \mathcal{W}^{\ell-|\alpha+\beta|} \partial_{\beta^{\prime \prime}}^{\alpha^{\prime \prime}} f\right\|\right\},
\end{array}
$$

and

$$
\begin{aligned}
E_{2}=\min \{ & \left\|\phi_{a} \mathcal{W}^{\ell-|\alpha+\beta|} \partial_{\beta^{\prime}}^{\alpha^{\prime}} f\right\|\left(\sup _{a^{\prime}}\left\|\left(1-\Delta_{x}\right) \phi_{a^{\prime}} \mathcal{W}^{4} \partial_{\beta^{\prime \prime}}^{\alpha^{\prime \prime}} f\right\|\right), \\
& \left.\left(\sup _{a^{\prime}}\left\|\left(1-\Delta_{x}\right) \phi_{a^{\prime}} \mathcal{W}^{\ell-|\alpha+\beta|+2} \partial_{\beta^{\prime}}^{\alpha^{\prime}} f\right\|\right)\left\|\phi_{a} \mathcal{W}^{2} \partial_{\beta^{\prime \prime}}^{\alpha^{\prime \prime}} f\right\|\right\} .
\end{aligned}
$$

Since $E_{1}, E_{2}$ are the same as $D_{2}, D_{3}$ in the section 3, respectively, we have

$$
\begin{gathered}
\left\|\phi_{a} \mathcal{W}^{\ell-|\alpha+\beta|} Q_{\varepsilon}^{+}\left(\partial_{\beta^{\prime}}^{\alpha^{\prime}} f, \partial_{\beta^{\prime \prime}}^{\alpha^{\prime \prime}} f\right)\right\|_{L^{2}\left(\mathbb{R}^{6}\right)} \lesssim\|f\|_{\mathcal{H}_{u l}^{k, \ell}}^{2} \\
\text { if }\left|\alpha^{\prime}+\alpha^{\prime \prime}\right|+\left|\beta^{\prime}+\beta^{\prime \prime}\right| \leq|\alpha+\beta| .
\end{gathered}
$$

Now we consider the following special case:

$$
\alpha+\beta=\alpha^{\prime}+\beta^{\prime}+\alpha^{\prime \prime}+\beta^{\prime \prime}+\alpha^{\prime \prime \prime}+\beta^{\prime \prime \prime}, \quad\left|\alpha^{\prime \prime \prime}+\beta^{\prime \prime \prime}\right| \geq k-1,
$$

and write $\partial^{q_{1}}, \partial^{q_{2}}=\partial_{\beta^{\prime}}^{\alpha^{\prime}}, \partial_{\beta^{\prime \prime}}^{\alpha^{\prime \prime}}$. Noting (5.2) with $a=x$, by applying Lemma 2.2 to both factors, we have

$$
\begin{aligned}
& \left\|\mathcal{W}^{\ell-|\alpha+\beta|} Q_{\varepsilon}^{+}\left(\partial_{\beta^{\prime}}^{\alpha^{\prime}} f, \partial_{\beta^{\prime \prime}}^{\alpha^{\prime \prime}} f\right)\right\|_{L^{\infty}\left(\mathbb{R}_{x}^{3} ; L^{2}\left(\mathbb{R}_{v}^{3}\right)\right)} \lesssim \sum_{\left|q_{1}+q_{2}\right| \leq 1} \\
& \left(\sup _{a^{\prime}}\left\|\left(1-\Delta_{x}\right) \phi_{a^{\prime}} \mathcal{W}^{7} \partial^{q_{1}} f\right\|\right)\left(\sup _{a^{\prime}}\left\|\left(1-\Delta_{x}\right) \phi_{a^{\prime}} \mathcal{W}^{\ell-|\alpha+\beta|+2} \partial^{q_{2}} f\right\|\right) \\
& \lesssim\|f\|_{\mathcal{H}_{u l}^{k, \ell}}^{2}
\end{aligned}
$$

because $\ell-3 \geq 7$, and the fact that from $\left|q_{j}\right|+\left|\alpha^{\prime \prime \prime}+\beta^{\prime \prime \prime}\right| \leq|\alpha+\beta|$ it holds that

$$
\ell-|\alpha+\beta|+2 \leq \ell-\left(\left|q_{j}\right|+2\right)+4-\left|\alpha^{\prime \prime \prime}+\beta^{\prime \prime \prime}\right| \leq \ell-\left(\left|q_{j}\right|+2\right)+(5-k) .
$$

Acknowledgements: The research of the first author was supported in part by Grant-in-Aid for Scientific Research No.25400160, Japan Society of the Promotion of Science. The research of the second author was supported in part by the General Research Fund of Hong Kong, CityU No.104511. The second author would also like to thank the National Center for Theoretical Sciences (Mathematics Division, Taipei Office) for the support and hospitalilty during his visit in July, 2013. 


\section{REFERENCES}

[1] R. Alexandre, L. Desvillettes, C. Villani, B. Wennberg, Entropy dissipation and long-range interactions, Arch. Ration. Mech. Anal. 152 (2000), 327-355

[2] R.Alexandre, Y.Morimoto, S.Ukai, C.-J.Xu and T.Yang, Uncertainty principle and kinetic equations, J. Funct. Anal., 255 (2008) 2013-2066.

[3] R. Alexandre, Y. Morimoto, S. Ukai, C.-J. Xu and T. Yang, Regularizing effect and local existence for non-cutoff Boltzmann equation, Arch. Rational Mech. Anal.,198 (2010), 39123.

[4] R. Alexandre, Y. Morimoto, S. Ukai, C.-J. Xu and T. Yang, Global existence and full regularity of the Boltzmann equation without angular cutoff, Comm. Math. Phys .,3-4-2(2011),513581.

[5] R. Alexandre, Y. Morimoto, S. Ukai, C.-J. Xu and T.Yang, Boltzmann equation without angular cutoff in the whole space: Qualitative properties of solutions, Arch. Rational Mech. Anal.,202(2011), 599-661.

[6] R. Alexandre, Y. Morimoto, S. Ukai, C.-J.Xu and T. Yang, Bounded solutions of the Boltzmann equation in the whole space, Kinetic and Related Models. 4 (2011) 17-40.

[7] R. Alexandre, Y. Morimoto, S. Ukai, C.-J. Xu and T. Yang, Uniqueness of solution for the non cutoff Boltzmann Equation with the soft potential, Kinet. Relat. Models, 4 (2011), 919-934.

[8] R. Alexandre, Y. Morimoto, S. Ukai, C.-J. Xu and T. Yang, Boltzmann equation without angular cutoff in the whole space: I, Global existence for soft potential, J. Funct. Anal. 262 (2012), 915-1010.

[9] Y. Morimoto, S. Ukai, C.-J. Xu, T. Yang, Regularity of solutions to the spatially homogeneous Boltzmann equation without angular cutoff. Discrete and Continuous Dynamical Systems Series A 24, 187-212(2009)

Y. Morimoto, Graduate School of Human and Environmental Studies, Kyoto UniVERSITY

КYOTO, 606-8501, JAPAN

E-mail address: morimoto@math.h.kyoto-u.ac.jp

T. Yang, Department of mathematics, City University of Hong Kong, Hong Kong, P. R. CHINA

AND

School of Mathematics, Wuhan University 430072, Wuhan, P. R. China

E-mail address: matyang@cityu.edu.hk 